\title{
Photostability of photosynthetic complexes coupled to silver nanowires
}

\author{
Magdalena Twardowska, Maria Olejnik, and Sebastian Mackowski \\ Optics of Hybrid Nanostructures Group, Institute of Physics, Faculty of Physics, Astronomy, and Informatics, \\ Nicolaus Copernicus University, Grudziadzka 5/7, 87-100 Toruń,
}

Received May 15, 2013; accepted June 25, 2013; published June 30, 2013

\begin{abstract}
We investigate the influence of plasmonic excitations in silver nanowires upon the photostability of a photosynthetic complex placed at their vicinity. Strong interaction between the two nanostructures is evident through an increase in the fluorescence intensity of the photosynthetic complexes close to the nanowires as compared to uncoupled ones and shortening of the fluorescence lifetime. The analysis of collected films, examined through a wide-field fluorescence microscope, with respect to temporal intensity variations for photosynthetic complexes located along and at the ends of the nanowires, indicates that the photostability is unaffected by the interaction with plasmonic excitations in metallic nanoparticles. Therefore, we conclude that in the case of studied hybrid nanostructures coupling with metallic nanoparticles results in a higher number of photons emitted from chlorophyll molecules.
\end{abstract}

Among photosynthetic complexes two general groups can be defined: reaction centers, which separate charges and therefore actively participate in the photochemistry of photosynthesis, and light-harvesting antennas, responsible for the absorption of sunlight. These complexes are typically pigment-protein assemblies, where the packing of pigments is strictly defined by the protein scaffold [1]. Recently, it has been shown that the absorption of lightharvesting complexes can be efficiently enhanced when coupled with plasmon excitations in metallic nanoparticles [2-6]. While, in a general case, the interaction with metallic nanoparticles influences both the absorption and emission rates [7], applying advanced techniques of sample fabrication has made it possible to selectively tune any of these two [2]. An important issue always related with coupling fluorophores to metallic nanoparticles concerns the photostability of emitters, in particular in the case where, as a result of the coupling, the radiative rate is strongly enhanced [8]. This effect is particularly important for biologically functional structures, such as lightharvesting complexes. While in the case of semiconductor nanocrystals it has been shown that the coupling to plasmons results in improved photostability [9], lightharvesting complexes deposited onto a silver island film exhibited increased photobleaching [10].

In this work we fabricate a hybrid nanostructure composed of chemically synthesized silver nanowires and peridinin-chlorophyll-protein (PCP) light-harvesting complexes from algae Amphidinium carterae. The monomer of PCP contains only two chlorophyll $a$ molecules embedded together with eight peridinins in a protein matrix [11]. The PCP complex, thanks to its wellunderstood optical properties, absorption spectrum located in the visible spectral range, strong fluorescence, and water solubility, is highly suitable as a model system for studying plasmonic interactions with functional biomolecules $[3,10]$.

The fluorescence properties of hybrid nanostructures were studied using wide-field fluorescence microscopy, a technique which allows for efficient acquisition of 100x100 micron large maps in sub-second intervals. A single image measured for the PCP complexes deposited onto a layer of silver nanowires exhibits well-resolved elongated shapes of high fluorescence intensity, which we attribute to the emission of the PCP complexes coupled to the silver nanowires. The intensity enhancement along the nanowire is not uniform and we observe that at the ends of the nanowires the intensity is higher than along them. The analysis of films collected for the sample enables us to extract the time dependence of fluorescence intensity of any given spot across the sample surface. We find that while the fluorescence lifetime of the PCP complexes coupled to the silver nanowires is shortened, the photobleaching remains unchanged, regardless of the position of the PCP complexes at the nanowires. Combining these two facts together implies that the PCP complexes emit a much larger number of photons when coupled to silver nanowires than being isolated from them. This result shows that metallic nanostructures -by improvement of their photostability - can significantly enhance the emitting properties of biologically functional nanostructures.

Silver nanowires (Ag NWs) were synthesized using a polyol process in the presence of ethylene glycol, copper seeds and poly(vinyl pyrrolidone) polymer. In the reaction ethylene glycol served as a reducing reagent and solvent [12]. The PCP complexes were reconstituted as previously described [13]. Hybrid nanostructures composed of silver nanowires and the PCP complexes were fabricated on glass coverslips using a spin-coating technique. In the first step we spin-coated $30 \mu \mathrm{L} \mathrm{Ag} \mathrm{NWs,} \mathrm{and} \mathrm{then} \mathrm{a} \mathrm{layer} \mathrm{of}$ 
$20 \mu \mathrm{L}$ PCP in 2\% polyvinyl alcohol (PVA from Sigma Aldrich) aqueous solution was deposited. In addition, a reference sample was fabricated by spin-coating $30 \mu \mathrm{L}$ PCP in aqueous PVA solution. During the sample preparation particular care was taken to avoid contamination with any impurities, the glass cover slips, on which the solutions were spin-coated were cleaned thoroughly using Hellmanex (Hellma Analytics).
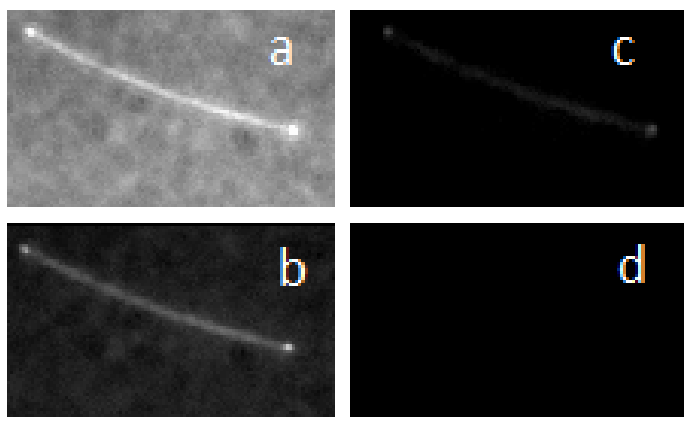

Fig. 1. Fluorescence images of a single silver nanowire covered with a layer of the PCP complexes at (a) $t=0 \mathrm{~min}$, (b) $t=5 \mathrm{~min}$, (c) $\mathrm{t}=10 \mathrm{~min}$, and (d) $t=15 \mathrm{~min}$. An excitation wavelength of $405 \mathrm{~nm}$ was used.

Fluorescence intensity maps were measured using a Nikon Eclipse $\mathrm{Ti}$ inverted wide-field microscope equipped with an Andor iXon Du-888 EMCCD detector. Due to the strong plasmon excitation of Ag NWs near 400 $\mathrm{nm}$ and the PCP absorption maximum near $480 \mathrm{~nm}$, LEDs illuminators with wavelengths of 405 and $480 \mathrm{~nm}$ were used as excitation sources. The excitation light was reflected to the microscope objective lens (Plan Apo, 100x, oil immersion, Nikon) by using dichroic beam splitters (Semrock FF458-Di02; Chroma 505DCXR). The excitation power after the beam splitter was $145 \mu \mathrm{W}$ and $60 \mu \mathrm{W}$ for the $405 \mathrm{~nm}$ and $480 \mathrm{~nm}$ excitation, respectively. The fluorescence of the PCP complexes coupled to the silver nanowires was filtered using a narrow band pass filter (Chroma HQ675-20) and detected as 1024×1024 pixel maps Typically, the gain of 50 was used for collecting the images with acquisition time of $5 \mathrm{sec}$.

In Fig. 1 we show fluorescence images extracted from a movie collected over 15 minutes for a single, wellresolved silver nanowire covered with a layer of the PCP complexes. The images obtained at (a) $\mathrm{t}=0 \mathrm{~min}$, (b) $\mathrm{t}=5$ min, (c) $t=10 \mathrm{~min}$, and (d) $\mathrm{t}=15 \mathrm{~min}$ are shown, and the intensity scale is the same for all the images. The first image shows an elongated shape of enhanced fluorescence emission as compared with the background. By correlating the fluorescence image with the one obtained in a transmission mode, we attribute the enhanced fluorescence intensity to the emission of the PCP complexes located in the vicinity of the silver nanowire. In addition to fluorescence enhancement along the nanowire, the fluorescence at the ends of the nanowire is even brighter. The appearance of much higher fluorescence intensity at the ends of the nanowires seems to be a generic effect, independent of the excitation wavelength [6]. This increase in fluorescence intensity can be due to a strong antennae effect combined with increased scattering of plasmon excitations at the ends of nanowires. Furthermore, time-resolved experiments indicate that the enhancement is mainly a result of increased radiative rate in the photosynthetic complexes [6], as the decay time for the PCP coupled to silver nanowires is approximately $1 / 10$ of the value measured for isolated PCP complexes.

The sequence of fluorescence images features a gradual decrease in fluorescence intensity of the PCP complexes in the structure, both of those located in the vicinity of the nanowires and away from them. Finally, after approximately 15 minutes there is very little emission coming off the sample.
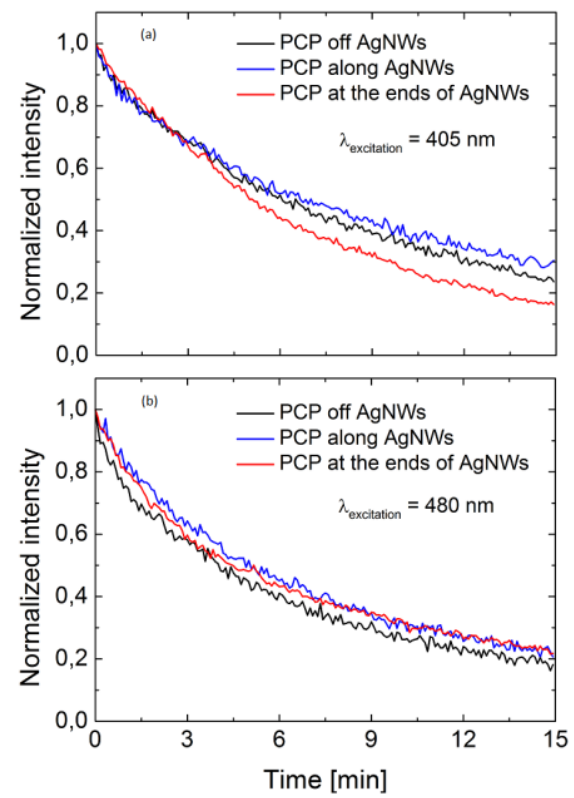

Fig. 2. Time evolution of the fluorescence intensity measured away from the nanowires (black), along the nanowires (blue), and at the ends of nanowires (red). For comparison, the results obtained for the PCP complexes excited with (a) $405 \mathrm{~nm}$ and (b) $480 \mathrm{~nm}$ are shown.

The evaluation of temporal behaviour of fluorescence intensity was carried out for individual pixels, corresponding to the emission at the ends of nanowires, along the nanowires and from the PCP complexes away from the nanowires. In Fig. 2 we show the results of such an analysis obtained for excitation wavelengths of (a) $405 \mathrm{~nm}$ and (b) $480 \mathrm{~nm}$. In contrast to previous work, where the fluorescence of the PCP complexes deposited on a silver island film was investigated and where the interaction with plasmon excitations resulted in significant decrease of photostability of the light-harvesting 
complexes, in the case of hybrid nanostructures studied in this work, the photobleaching shows no dependence upon the presence or absence of plasmonic interactions. There are also no significant differences between both excitation wavelengths apparent.

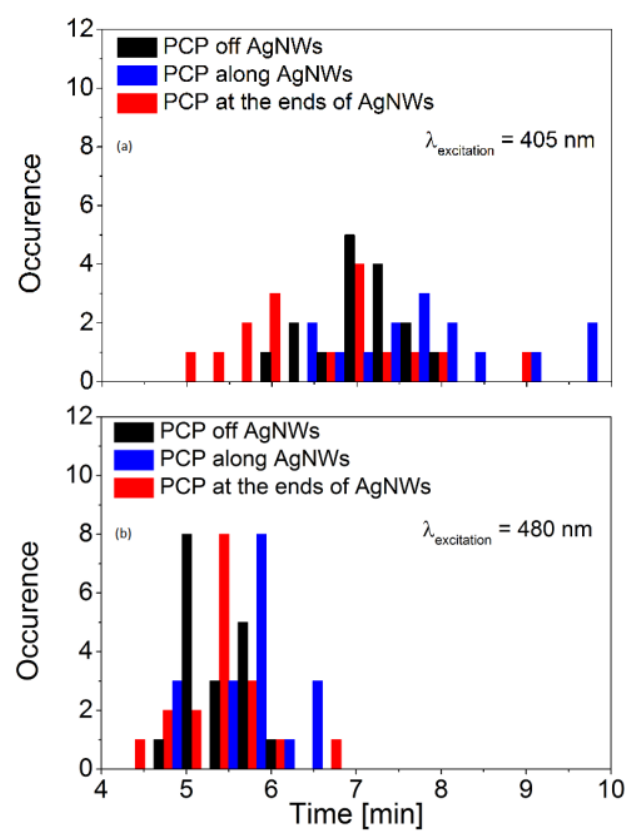

Fig. 3. Histograms of photobleaching constants determined form timetraces similar to the ones shown in Fig. 2. The results obtained for the PCP complexes away from the nanowires (black), along the nanowires (blue), and at the ends of nanowires (red) are shown. We also compare the values obtained for the PCP complexes excited with (a) $405 \mathrm{~nm}$ and (b) $480 \mathrm{~nm}$.

In order to gain a statistically relevant insight into the influence of plasmonic excitations in the silver nanowires on the photostability of the PCP fluorescence, we carried out a similar analysis for approximately 30 locations of each kind. The result of this analysis is displayed in Fig.3. While the differences observed for a given excitation wavelength between coupled and uncoupled PCP complexes are minute, the photostability of the PCP emission excited with $405 \mathrm{~nm}$ is significantly better than in the case of $480 \mathrm{~nm}$ excitation. The fluorescence lasts approximately $50 \%$ longer in this case, regardless of over twofold higher excitation power.

In conclusion, we find that the fluorescence photostability of photosynthetic complexes remains unaffected upon coupling with plasmonic excitations in silver nanowires. A detailed analysis of temporal evolution of the fluorescence intensity for the PCP complexes located along and at the ends of nanowires yields no significant differences in their photobleaching as compared to isolated PCP complexes. In conjunction with much faster decay of emission this result indicates that the PCP complexes coupled to plasmon excitations in the silver nanowires emit a much higher total number of photons. This observation can have implications into possible design of functional hybrid nanostructures involving biologically relevant complexes as well as efforts aimed at controlling light properties using plasmonic nanostructures.

The research was supported by the WELCOME project "Hybrid Nanostructures as a Stepping Stone towards Efficient Artificial Photosynthesis" funded by the Foundation for Polish Science and EUROCORES project "BOLDCATS" funded by the European Science Foundation. We thank Eckhard Hofmann (University of Bochum, Germany) for reconstituted PCP complexes, and Nikodem Czechowski (Nicolaus Copernicus University, Poland) for providing software for timetrace evaluation.

\section{References}

[1] R.E. Blankenship, Wiley-Blackwell, ISBN: 978-0-632-04321-7 (2002).

[2] Ł. Bujak, N. Czechowski, D. Piatkowski, R. Litvin, S. Mackowski, T.H.P. Brotosudarmo, R.J. Cogdell, S. Pichler, W. Heiss, Appl. Phys. Lett. 99, 173701 (2011).

[3] N. Czechowski, P. Nyga, M.K. Schmidt, T.H.P. Brotosudarmo, H. Scheer, D. Piatkowski, S. Mackowski, Plasmonics 7, 115 (2012).

[4] J.B. Nieder, R. Bittl, M. Brecht, Angew. Chem. Int. Ed. 49, 10217 (2010).

[5] A. O. Govorov, I. Carmeli, Nano Letters 7, 620 (2007).

[6] M. Olejnik, B. Krajnik, D. Kowalska, M. Twardowska, N. Czechowski, E. Hofmann, S. Mackowski, Appl. Phys. Lett. 102, 083703/1 (2013).

[7] P. Anger, P. Bharadwaj, L. Novotny, Phys. Rev. Lett. 96, 113002 (2006).

[8] J.R. Lakowicz, Analytical Biochemistry 298(1), 1 (2001)

[9] K. Ray, R. Badugu, J.R. Lakowicz, J. Am. Chem. Soc. 128, 8998 (2006).

[10] S. Mackowski, S. Wörmke, A.J. Maier, T.H.P. Brotosudarmo, H. Harutyunyan, A. Hartschuh, A.O. Govorov, H. Scheer, C. Bräuchle, Nano Letters 8, 558 (2008).

[11] A.D. Ferguson, E. Hofmann, J. W. Coulton, K. Diederichs, W. Welte, Science 282, 2215 (1998).

[12] K.E. Korte, S.E. Skrabalak, Y. Xia, J. Mater. Chem. 18, 437 (2008).

[13] T.H.P. Brotosudarmo, E. Hofmann, R.G. Hiller, S. Wörmke, S. Mackowski, A. Zumbusch, C. Bräuchle, H. Scheer, FEBS Letters 580, 5257 (2006) 